Musées, Patrimoine et Culture scientifiques et techniques

$173 \mid 2017$

septembre-octobre 2017

\title{
Les enjeux de la diversité : pour une médiation multiculturelle
}

\section{Saoussan Sabeh}

\section{OpenEdition}

\section{Journals}

Electronic version

URL: http://journals.openedition.org/ocim/1849

DOI: 10.4000/ocim.1849

ISSN: 2108-646X

\section{Publisher}

OCIM

\section{Printed version}

Date of publication: 1 September 2017

Number of pages: 12-19

ISSN: 0994-1908

\section{Electronic reference}

Saoussan Sabeh, «Les enjeux de la diversité : pour une médiation multiculturelle », La Lettre de l'OCIM [Online], 173 | 2017, Online since 01 September 2018, connection on 10 December 2020. URL : http:// journals.openedition.org/ocim/1849; DOI : https://doi.org/10.4000/ocim.1849

This text was automatically generated on 10 December 2020.

Tous droits réservés 


\title{
Les enjeux de la diversité : pour une médiation multiculturelle
}

\author{
Saoussan Sabeh
}

"Bosou" et "Legba", par Dubréus Lhérisson, présentés à l'exposition Haïti. Deux siècles de création artistique au Grand Palais à Paris du 19 novembre 2014 au 15 février 2015. Auriez-vous reconnu ces sculptures prophylactiques d'inspiration vaudou sans légende, ni cartel ?

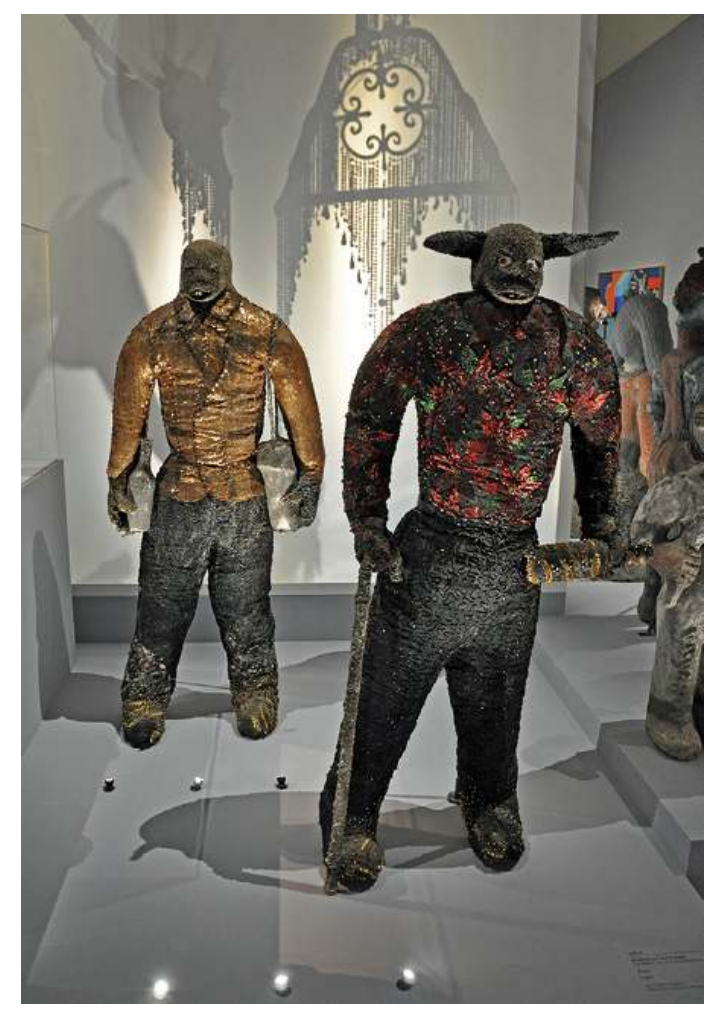

(c) Saoussan Sabeh

1 La loi Musée du 4 janvier 2002 fut la première en France à officialiser, légalement parlant, le rôle pédagogique et hédoniste de l'institution muséale ${ }^{1}$. La médiation constitue ce 
mode de communication spécifique que les musées se doivent d'établir avec leur public, dans le but de favoriser la compréhension, le plaisir et l'apprentissage du plus grand nombre.

Or, le public représente aujourd'hui une entité très diversifiée : touristes d'origine étrangère, population locale native, immigrés... En outre, nombre de musées conservent des objets venus d'horizons - historiques ou géographiques - plus ou moins lointains. Rendre ces expôts compréhensibles à "tous" suppose en réalité de s'adapter à chacun œuvre et visiteur - de manière subtile, adéquate et équitable.

\section{Le multiculturalisme des objets}

3 L'indéniable diversité qui caractérise tant le public que les collections des institutions muséales impose au milieu professionnel un multilinguisme, mais surtout un multiculturalisme patent, pourtant méconnu et écarté de la plupart des politiques muséales. Tous les musées ne ciblent certes pas le public touristique, mais aucun d'eux ne lui est évidemment défendu. Dans le cadre d'un établissement muséal situé en zone frontalière par exemple, le public local est déjà un public plurilingue et pluriculturel, sans qu'il soit besoin pour cela de considérer le public touristique. En outre, si la France considère la seule langue française comme officielle, cet exemple ne vaut pas pour toutes les nations. Ses voisins limitrophes possèdent pour plusieurs d'entre eux divers idiomes officiels : ainsi l'Espagne, la Belgique ou la Suisse. Seuls $7 \%$ des institutions interrogées dans le cadre de l'enquête se contentent d'ailleurs d'une offre de médiation unilingue. Le multilinguisme, déjà intégré par une grande partie du milieu professionnel comme une donnée fondamentale pour l'accessibilité du public dans son ensemble, doit donc être perpétué et encouragé, mais c'est le multiculturalisme surtout qu'il convient aujourd'hui d'intégrer aux pratiques professionnelles, en parallèle constant aux efforts linguistiques.

Chaque objet est façonné dans des matériaux propres à un environnement, selon les savoir-faire et les croyances spécifiques de la culture de l'artiste ou de l'artisan qui l'a créé. Chaque composante des collections muséales porte donc en elle une sémantique précise, liée à son contexte de création et enrichie par les divers contextes de sa réception au fil du temps. La signification de l'objet peut différer de celle que lui donne le visiteur d'après ses propres codes culturels, aussi est-il nécessaire de "traduire" l'objet et ses contextes (de fabrication, d'usage, de réception...) au public afin de permettre à ce dernier de mieux l'appréhender, et de le lui rendre compréhensible.

5 Cette traduction ne peut fonctionner sans que ne soit également prise en compte la culture du visiteur ciblé, au sens de l'environnement de savoirs qui est le sien, c'est-à-dire ses connaissances, les mœurs de la société dans laquelle il évolue, les traditions de son lieu de naissance et/ou de résidence... qui fondent son identité. En effet, la culture entendue comme l'ensemble des savoirs et des us propres à chaque groupe humain influence et oriente la compréhension des visiteurs.

\section{Lorsqu'incompréhensible ne rime pas qu'avec exotique}

Le multiculturalisme s'entend plus aisément lorsque l'on considère des objets "exotiques" au sens premier du terme, tels qu'envisagés par rapport à un environnement de savoirs de référence, comme le seraient des masques funéraires d'Océanie appréhendés par un 
public européen, par exemple. Pourtant, la distance géographique est loin de constituer l'unique barrière à la compréhension des visiteurs : l'éloignement temporel, ou religieux, ou social ou culturel, y compris dans un même pays ou une même région, demeure une source de questionnements, même pour des œuvres issues du référentiel culturel du public considéré. Ainsi, nombre de retables ont perdu sur les cimaises des musées l'aura religieuse qui faisait leur valeur à l'époque de leur création, pour n'être plus observés aujourd'hui qu'au travers de leurs qualités esthétiques. Quelle proportion des visiteurs s'avère capable de nos jours d'identifier les codes iconographiques mythologiques ou bibliques ? Le multiculturalisme est devenu une nécessité pour concevoir et mettre en œuvre des outils de médiation pertinents pour l'ensemble des visiteurs. Contrairement aux idées reçues, l'adaptation des aides à la visite ne vaut pas uniquement pour un public étranger, ou immigré, ou "du champ social". La majorité du public muséal se compose de primo-visiteurs ; or la plupart ignore les clefs de lecture (codes iconographiques, repères historiques et géographiques...) qui leur permettraient d'appréhender, au moins en partie, les œuvres qui leur sont proposées. Le système scolaire ne fournit plus, ou peu, ces éléments. Il revient donc aux musées de pallier ces difficultés en éclairant la lecture de leurs collections, en prenant en compte l'environnement de savoirs des visiteurs et le contexte des œuvres quels qu'ils soient, sans tomber, comme c'est encore trop souvent le cas, dans un ciblage condescendant qui ne viserait que certaines populations ou certains objets, plus sujets que d'autres à favoriser l'incompréhension dans l'imaginaire collectif.

Une proportion non négligeable de visiteurs ignore en réalité quel chapitre de l'histoire biblique est représenté par Fra Angelico dans Le Couronnement de la Vierge (musée du Louvre, Paris).

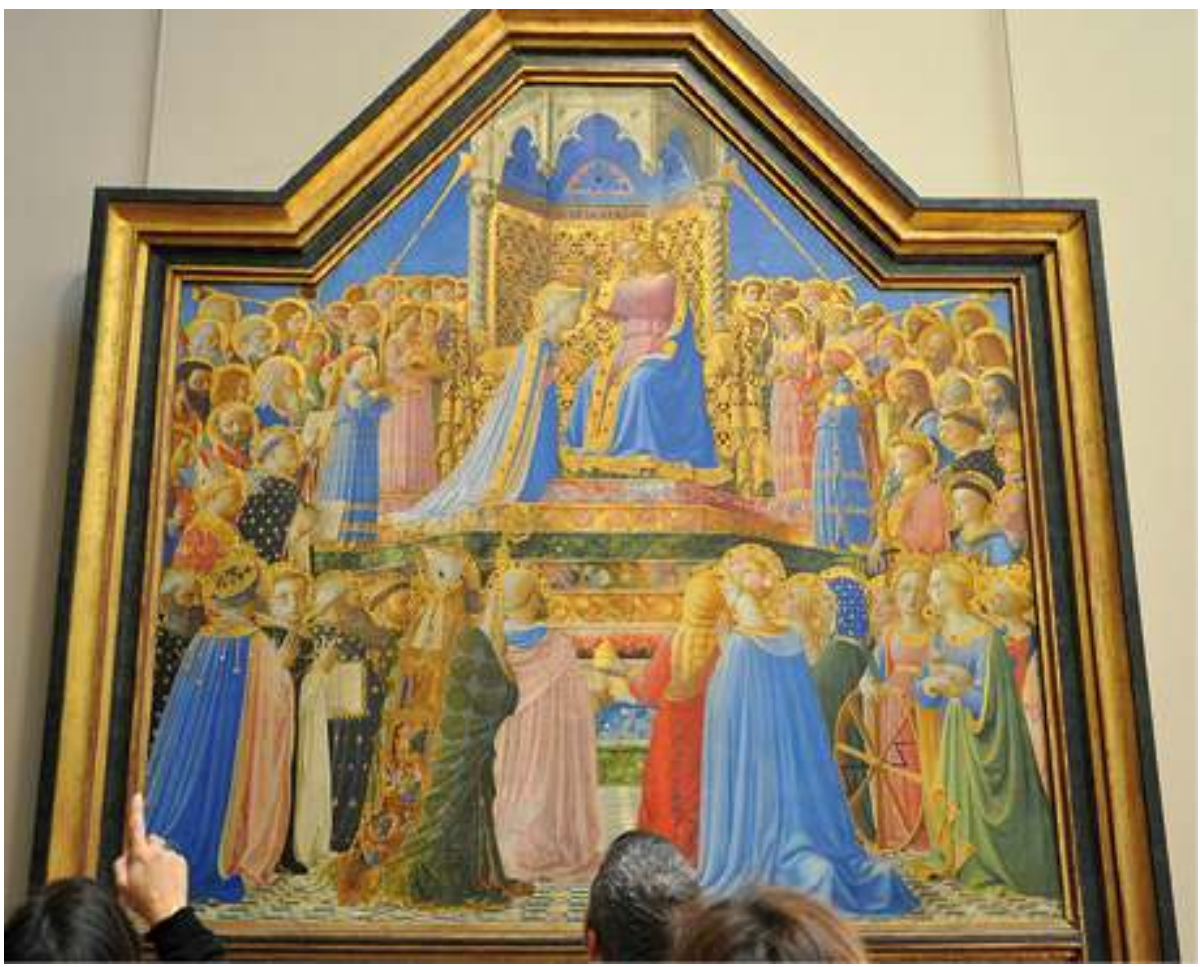

(c) Saoussan Sabeh 


\section{Diversité du public et multilinguisme}

7 Le public des musées est aujourd'hui très varié. Les institutions ne peuvent se baser sur le seul critère géographique pour cerner la culture (au sens de références et de connotations communes aux membres d'un même groupe humain et/ou ethnique et propres à chacun d'eux) de leurs visiteurs. Le public de proximité, le public touristique par exemple recouvrent chacun des entités très diverses culturellement parlant : les touristes peuvent être nationaux ou étrangers ; le public de proximité, quant à lui, peut être natif ou "d'adoption" (nationaux et/ou immigrés résidents), et appartenir (ou non) au public du champ social. Il peut également désigner les communautés d'origine du patrimoine conservé, qu'il s'agisse de populations "déplacées" (immigrés arabes ou maghrébins habitant Paris pour les œuvres présentées au département des arts de l'Islam du musée du Louvre) ou non (œuvres aborigènes exposées dans les musées australiens). Cette complexité peut s'accentuer si l'institution s'inscrit dans un territoire à l'identité culturelle forte voire distincte de l'identité nationale, comme c'est le cas en France dans certaines régions comme l'Alsace, la Bretagne, la Corse ou encore le Pays basque. L'adaptation culturelle s'avère très exigeante car elle oblige à prendre en compte, non seulement les particularités de l'œuvre, mais aussi les cultures nationales voire régionales du public.

8 Le multilinguisme appliqué aux aides à la visite ne peut donc pas suffire à une réelle et durable compréhension par le public. Si la traduction constitue un premier niveau d'adaptation, elle n'en demeure pas moins une démarche, certes évidente, mais basique dans l'accomplissement d'une communication muséale aboutie entre les institutions et leurs visiteurs. En effet, la traduction est une nécessité au vu de l'augmentation des flux migratoires, car le public parle aujourd'hui des langues très variées. Pour certains auteurs, c'est justement ce besoin de traduire les aides à la visite dans des idiomes divers qui justifie la nécessité de leur adaptation culturelle. En effet, la langue reflète son environnement, ainsi que la vision du monde qui lui correspond, par le vocabulaire qu'elle attribue aux différents éléments qui les composent, par exemple. La langue reflète donc la culture, et pour bien traduire linguistiquement, il faut également adapter culturellement, car toutes les notions n'existent pas dans toutes les cultures sous les mêmes auspices. Tout l'implicite d'un énoncé doit être traduit culturellement pour être compris de tous, puisque références et connotations divergent selon les pays, les religions, les langues et les cultures.

\section{Vacuité bibliographique et manque de sources}

En matière de multiculturalisme dans la médiation muséale, les sources bibliographiques disponibles s'intéressent surtout, et de plus en plus, au public du champ social issu de l'immigration et aux communautés d'origine du patrimoine conservé, dites "communautés-sources". 
Oreiller en forme de tigre et son cartel unilingue, musée national des Arts asiatiques-Guimet (Paris). L'inconvénient de la traduction comme de l'adaptation des supports de médiation réside dans l'espace et/ou le volume muséal qu'elles nécessitent. Il est aisé d'imaginer la masse textuelle que représenterait la traduction de ce simple cartel dans deux autres langues, encore démultipliée si le processus était appliqué à l'ensemble de la vitrine.

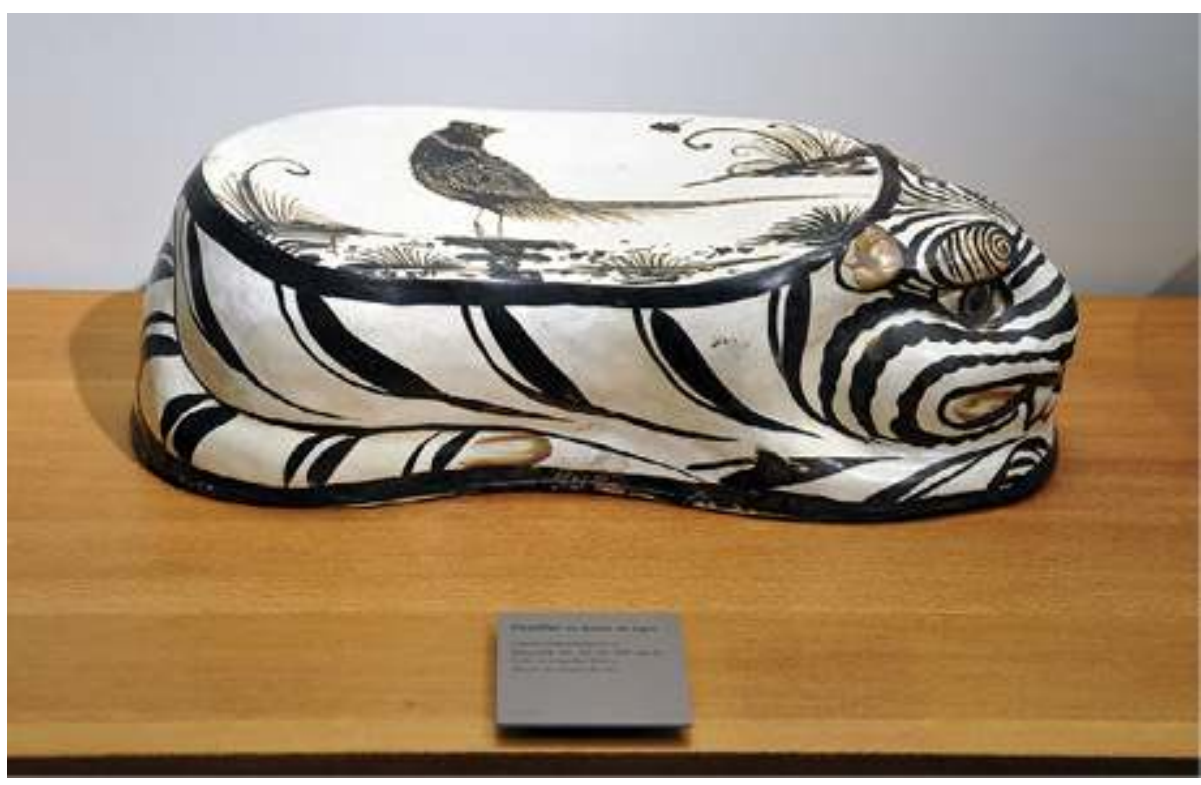

(C) Saoussan Sabeh

10 Très rares sont les ouvrages, revues ou articles permettant de s'immerger dans les modalités concrètes de l'adaptation telle que décrite ici. À quelques exceptions près, la plupart des sources se consacrent à la diversité culturelle dans ses considérations abstraites, mettant notamment en garde contre la difficulté d'établir et d'entretenir un équilibre entre préservation d'identités différentes et création d'un dialogue pacifique et harmonieux entre ces identités, au sein d'une même société.

11 La littérature aborde peu la traduction des outils de médiation, pourtant favorisée lorsqu'on la compare aux questions culturelles liées à l'adaptation des aides à la visite. Il en résulte aujourd'hui un déséquilibre bibliographique flagrant, en faveur bien entendu des aspects linguistiques de la médiation.

\section{Des notions délicates à manier}

De telles lacunes dans les sources muséologiques reflètent probablement l'appréhension des auteurs, chercheurs comme professionnels, à se confronter aux considérations concrètes portées par la diversité culturelle. Semblables notions s'avèrent extrêmement délicates, voire risquées, à manier, comme le prouvent les débats continuels qu'elles suscitent dans la vie publique.

13 Ces réticences et le malaise qu'elles dissimulent ne doivent pas écarter le refus pur et simple d'une démarche d'adaptation des outils de médiation, parce qu'inutile (du moins considérée comme telle) ou allant à l'encontre des méthodes traditionnelles (simples traductions linguistiques), suffisantes pour certains. La vacuité bibliographique en la matière explique que l'adaptation en question demeure inconnue dans nombre 
d'institutions muséales et auprès de la plupart des professionnels, dont il est également fort probable qu'ils n'aient simplement pas envisagé d'initier une telle démarche.

Celle-ci se révèle d'autant plus délicate à mettre en œuvre que le musée se veut un lieu neutre et objectif. Institution de référence pour découvrir l'Autre aussi bien que Soimême, l'établissement muséal ne doit en aucun cas devenir un instrument de propagande visant à propager un point de vue particulier au détriment de la vérité. Ce positionnement ne doit évidemment pas mener à l'excès inverse par la censure d'œuvres ou de faits qui pourraient déplaire à certains publics, au risque d'emprisonner ceux-ci dans leur méconnaissance et leurs préjugés. La neutralité scientifique doit être conservée, car, outre qu'elle constitue le plus sûr gage de l'excellence de l'institution, elle garantit également la véracité de ce que le musée expose et transmet aux visiteurs. L'adéquation entre adaptation culturelle et neutralité scientifique pose la question du degré d'adaptabilité dont doivent faire preuve les musées, pour que leur objectivité n'entame pas leur mission pédagogique, et inversement. Certaines religions déprécient par exemple la nudité, qui caractérise pourtant nombre d'œuvres phares de l'histoire de l'art. L'objectivité du musée, sa neutralité demande que semblables expôts soient présentés à l'ensemble des visiteurs, sans que les choix de leur auteur ne soient ni encensés ni condamnés, mais simplement expliqués. L'adaptation culturelle doit permettre de contourner ces réticences et d'expliciter les œuvres selon les références du public afin qu'elles deviennent accessibles à, et compréhensibles par tous. L'adaptation doit également permettre d'éviter que les expôts ne deviennent le support ni l'objet de préjugés et d'erreurs.

La diversité culturelle soulève les mêmes interrogations dans la société comme au sein de l'institution muséale, à savoir comment considérer l'Autre, et quelle place lui donner, sans se renier Soi-même ? La prise en compte des cultures dans la médiation muséale reflète les débats de l'actualité contemporaine, qui reconnaît la diversité comme une richesse mais peine à assurer l'harmonie entre les différentes identités existantes, qu'elles soient nationales, régionales et/ou minoritaires. Les difficultés rencontrées coïncident avec les craintes de chaque culture de se voir effacée ou dominée par une autre. Le musée, par le patrimoine qu'il conserve et présente au public et par ses actions de médiation auprès des visiteurs, pourrait devenir le lieu de leur réconciliation, ou du moins de leur compréhension mutuelle.

\section{Prise en compte des enjeux linguistiques et culturels dans la conception des aides à la visite}

16 Les recherches engagées en 2016 par l'auteur ${ }^{2}$ dans le cadre d'un travail universitaire s'appuient sur les contributions d'une soixantaine d'institutions muséales, françaises pour la plupart mais également étrangères, pour éclairer les modalités concrètes de l'adaptation culturelle des aides à la visite et les difficultés de sa mise en place.

La démarche étudiée s'inscrit rarement dans la politique officielle des musées : elle ne participe dans $74 \%$ des $\operatorname{cas}^{3}$ ni des objectifs des établissements ni des orientations impulsées par la hiérarchie ou le PSC. Pourtant, l'adaptation des outils de médiation correspond au ciblage des visiteurs tel que la plupart des institutions $(78 \%)$ le conçoivent, à savoir principalement d'après un critère géographique (qui différencie public de proximité et touristes). Ce critère distinctif induit, de manière plus ou moins implicite, 
des aspects linguistiques et culturels. Alors que la nécessité de la traduction a globalement été intégrée par la majorité des musées, ceux-ci peinent encore, semble-t-il, à appréhender les enjeux de l'adaptation culturelle des aides à la visite, d'autant que les flux numériques et médiatiques liés aux mouvements de mondialisation laissent facilement croire à l'émergence d'une culture "globalisée" partagée par tous.

Cartel unilingue présenté à l'exposition Céramiques surprises. Faïences et trompe-l'œil des XVIII'e et $X I X e$ siècles (musée départemental de la Faïence et des Arts de la table, Samadet). S'il s'avère difficile de multiplier sur les cimaises les panneaux et les cartels à destination de différentes langues et cultures, l'utilisation d'autres supports de médiation comme les fiches de salle ou les audioguides permettrait de pallier ces difficultés. Bien entendu, chaque aide à la visite proposée au public, même unilingue, impose des coûts de fabrication, bien que les plus onéreuses ne soient pas nécessairement les plus pertinentes.

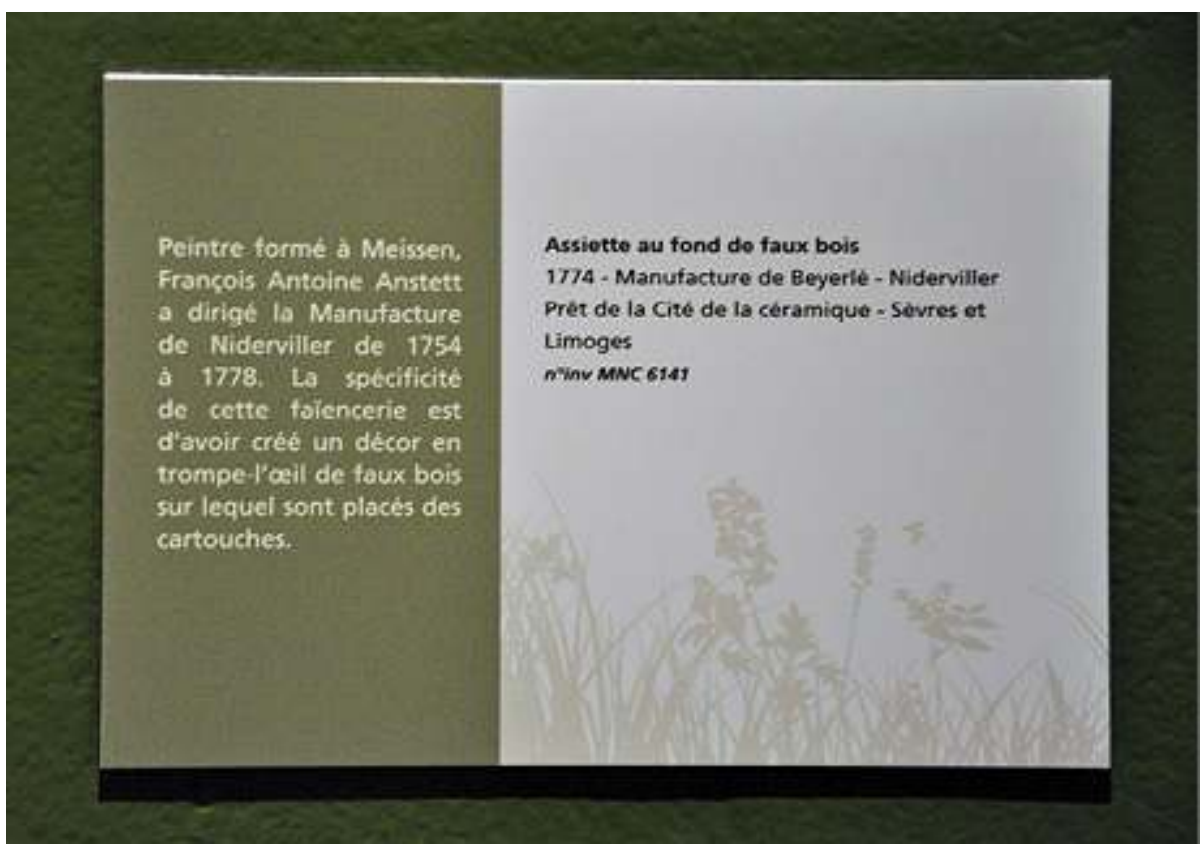

(c) Saoussan Sabeh

En effet, $53 \%$ des institutions interrogées proposent des aides à la visite multilingues (quatre idiomes ou plus). Seuls $7 \%$ des établissements sollicités, tous français (indice symptomatique ?) s'avèrent unilingues. Les résultats de l'enquête confortent l'anglais comme "langue internationale" par excellence, puisque présente dans $98 \%$ des établissements, en France comme à l'étranger. Les idiomes régionaux ou minoritaires, quant à eux, demeurent les parents pauvres de l'offre linguistique française, bien qu'ils soient proposés dans au moins $50 \%$ des établissements situés dans des territoires pourvus d'une identité linguistique et culturelle forte, distincte de celles de la Nation. Semblables inégalités de traitement entre les langues reflètent très probablement les tensions internes au pays, relatives au développement des langues (et implicitement, des cultures) régionales. Certains territoires, comme l'Alsace, la Bretagne, le Pays basque ou la Corse, forts d'une culture et d'un idiome propre, s'attachent à défendre ceux-ci face à une politique nationale assez frileuse quant à la valorisation de ces identités, différentes quoique constitutives de la sienne. Les comptes-rendus des débats passionnés qui se succèdent depuis plusieurs années au Parlement, et notamment au Sénat, montrent à quel point ces questions recèlent d'interrogations, de craintes et d'espoirs ${ }^{4}$. 
19 L'effort de traduction ou d'adaptation des aides à la visite souligne la considération de l'institution muséale envers son public. Un véritable rapport de force s'exerce à travers les choix de l'établissement pour son public, soit qu'il lui offre un accès facilité aux expôts, soit qu'il lui en interdit la compréhension. Les taux précédemment énoncés montrent que la plupart des musées ont intégré et compris la nécessité d'une offre linguistique variée, étant donnée la diversité du public actuel. C'est un paradoxe que de constater que, si entendement linguistique (compréhension des individus entre eux grâce à l'usage d'un même langage) et entendement intellectuel (compréhension des expôts par le public) vont de pair et sont reconnus comme tels par les institutions muséales, entendement culturel (compréhension des individus entre eux grâce à l'usage de références et de connotations communes) et entendement intellectuel ne semblent pas influer l'un sur l'autre aux yeux de ces mêmes établissements. La pertinence de l'adaptation culturelle des aides à la visite semble en revanche mieux comprise lorsqu'elle s'intègre dans un contexte de diversité : dans des régions à forte identité culturelle et/ou linguistique, distincte de l'identité nationale, ou dans des lieux de brassage de populations comme l'est la capitale parisienne, par exemple. C'est d'ailleurs dans ces territoires qu'elle est le plus pratiquée, en France.

\section{Statut de la médiation et considération du public : synonymes?}

20 Contrairement à ce que pourrait laisser croire l'offre muséale française telle que proposée majoritairement à l'heure actuelle, les traductions et les adaptations sont attendues par le public. Aucune des institutions interrogées n'a fait part de réactions négatives des visiteurs envers l'une ou l'autre de ces démarches, mais bien au contraire de leurs demandes en la matière (58 \%), lorsqu'ils n'en disposent pas, ou de leur satisfaction (45\%) lorsque ces méthodes sont effectivement suivies ${ }^{5}$.

21 Le statut de la médiation pourrait ne pas être étranger au décalage existant entre l'offre muséale et les besoins du public. Il semblerait en effet que ce domaine muséologique ne bénéficie pas de la même image ni de la même valeur scientifique dans le milieu professionnel que d'autres, comme l'étude des collections ou la restauration. La considération qui leur est accordée influe forcément sur la qualité des supports de médiation, et reflète également l'attention que l'institution porte à ses visiteurs. Un semblant d'équilibre statistique a pu être constaté entre les établissements menant de véritables études scientifiques sur leurs aides à la visite et ceux privilégiant "l'expérience au quotidien", bien que cette dernière tendance soit la plus forte (56 \% contre $44 \%$ ). Nombre de musées ne possèdent pas les moyens financiers de réaliser des enquêtes de publics, ni de mettre en œuvre des outils numériques de médiation, d'autant que la pertinence pédagogique et/ou hédoniste de ces derniers n'est pas prouvée. Pourtant, $74 \%$ des institutions sollicitées envisagent de faire évoluer leur offre de médiation vers les nouvelles technologies, au risque d'investir de fortes sommes dans des supports exposés à une maintenance coûteuse et à une obsolescence rapide, sans que les bénéfices pour le public (et par voie de conséquence, pour l'institution) n'en soient explicités, ni même avérés. Le paragraphe suivant souligne que le manque de volonté n'est pas le seul obstacle au développement de la médiation (et des études qui lui sont liées). Inversement, son coût à lui seul n'en constitue pas forcément un non plus, puisque certains des établissements pratiquant l'adaptation culturelle ne correspondent pas à de "grandes" 
institutions. La thématique des musées n'induit aucune discrimination dans les résultats constatés de l'enquête, ni dans la mise en œuvre de telle ou telle méthode de médiation. Le panel de l'étude est très divers : tous les établissements patrimoniaux qui souhaitèrent apporter leur contribution furent pris en compte. Y figurent donc au moins : 3 musées étrangers, 7 musées parisiens, 11 "petits" établissements, 9 "grands" établissements, 4 institutions liées à la nature, 7 monuments ou sites historiques, 2 établissements liés à l'écrit et/ou à la littérature, 27 à l'art, 11 à l'histoire, 1 à la science, 2 à l'ethnographie...

Si la connaissance véritable du public, de ses attentes et de ses besoins dépend certes d'une pratique "de terrain", celle-ci devrait néanmoins être indissociable d'études poussées, dont le degré de scientificité conditionnerait la véracité des résultats. Ce dernier critère semble pourtant faire défaut dans l'exercice actuel de la médiation et dans la conception de cette dernière.

La retenue de certaines cultures envers la nudité ne doit pas influer sur la présentation au public d'œuvres comme le Faune endormi de Bouchardon (musée du Louvre, Paris). Le discours autour de cette sculpture, qui appartient aux collections publiques françaises, demande simplement à être adapté en fonction des visiteurs afin que tous puissent accéder aux clefs de lecture nécessaires à sa compréhension.

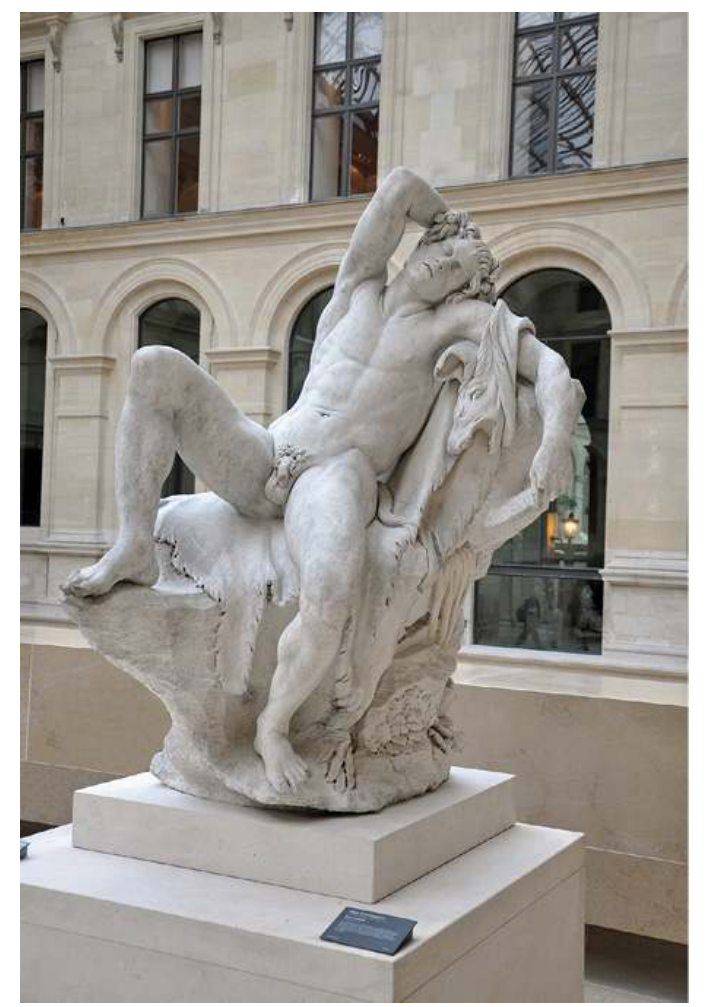

(c) Saoussan Sabeh

Ces lacunes ne sont pas le seul fait des professionnels de musée, mais aussi, malheureusement, du contexte économique défavorable que connaît aujourd'hui le monde de la culture et du patrimoine, en France du moins. Ces aspects financiers jouent sur les possibilités et les capacités des musées à adapter leurs outils de médiation aux visiteurs. Ils influencent également la volonté des structures patrimoniales, qui préfèrent parfois privilégier certains publics plus rentables. Les restrictions budgétaires ont ainsi des répercussions directes sur le développement des aides à la visite en langues régionales et/ ou minoritaires (et encore plus en ce qui concerne les cultures 
correspondantes, déjà ignorées aux échelles nationales), qu'elles interdisent plus ou moins puisque ces outils de médiation doubleraient les aides à la visite à destination d'un public qui bénéficie déjà de supports dans la langue nationale.

\section{Traduction ou adaptation : méthodes, différences et compromis}

littérale. L'adaptation culturelle serait pratiquée dans $42 \%$ des institutions sollicitées, en particulier pour ce qui a trait à la médiation humaine (guides). Cette adaptation est donc possible d'une part, et pertinente d'autre part, puisqu'elle est recherchée et mise en œuvre presque naturellement par les médiateurs. Il est vrai que l'interaction orale entre un guide et son groupe permet cette adaptabilité, qu'il peut être difficile d'instaurer sur les autres supports ; difficile, mais peut-être pas impossible. Les automatismes qu'entraîne le dialogue quant au choix du vocabulaire et du contenu du message posent la question de l'orientation, réelle ou vantée, des structures interrogées : les $42 \%$ cités auparavant représentent-ils des établissements où l'adaptation culturelle résulte d'un objectif instauré par les musées, ou simplement de la conscience professionnelle des médiateurs, relayée par les institutions sans que celles-ci n'en aient véritablement pris la mesure?

L'enquête a révélé l'existence d'une troisième possibilité, intermédiaire entre traduction et adaptation : les traductions "linguistiques". Celles-ci offrent une médiation de meilleure qualité qu'une traduction "mot-à-mot", car elles s'attachent à respecter les normes linguistiques de l'idiome de destination. Elles s'avèrent en revanche moins exigeantes que l'adaptation culturelle, puisqu'elles n'établissent pas de passerelles entre les différents environnements de savoirs. Les traductions linguistiques constituent des compromis de qualité, car elles proposent des aides à la visite plus satisfaisantes et mieux compréhensibles par le public que les traductions littérales, sans nécessiter les connaissances ni le budget que requiert l'adaptation culturelle. Pourtant, cette démarche s'est révélée être la moins répandue, puisqu'elle n'aurait été mise en œuvre que dans $9 \%$ des établissements sollicités.

Une dernière donnée quant au statut des traducteurs (qu'il s'agisse de traductions ou d'adaptations) pose enfin la question de la qualité scientifique des outils de médiation : en effet, les traducteurs les plus sollicités $(74 \%)$ sont en réalité des prestataires extérieurs ; le personnel interne n'arrive qu'en seconde position (cité par $58 \%{ }^{6}$ des institutions) dans le classement obtenu lors de l'enquête. Seuls $20 \%$ des structures font état d'une collaboration entre ces deux groupes (relecture des traductions externes par les membres de l'institution, échanges multiples lors de leur élaboration...). Quant aux traducteurs issus des communautés visées, quelles qu'elles soient, ils ne sont cités que par $12 \%$ des établissements interrogés. Ces chiffres pourraient laisser entendre que les compétences nécessaires à la traduction et à l'adaptation ne sont pas ou peu maîtrisées par le personnel des musées : les compétences linguistiques des Français (souvent moquées à l'étranger), et par là-même la formation éducative et scolaire en la matière, ont vraisemblablement une incidence sur la capacité des institutions patrimoniales françaises à traduire leur contenu, obligeant celles-ci à supporter le coût d'une délégation. En outre, l'externalisation des traductions permet aux professionnels de se soustraire à l'exercice 
de la médiation et de lui interdire en quelque sorte d'accéder à ses lettres de noblesse, puisqu'eux-mêmes ne la pratiquent pas, entretenant ainsi une distance peu souhaitable avec le public.

\section{L'adaptation culturelle en question : définition et mise en œuvre}

La crainte de catégoriser les visiteurs selon des connaissances et des sensibilités supposées semble le principal obstacle à une adaptation culturelle de la médiation. Certains des professionnels interrogés refusaient l'idée d'une adaptation des aides à la visite, qu'ils concevaient comme un choix de délivrer des informations différentes en fonction de stéréotypes.

Cette voie de réflexion se révèle effectivement totalement inadéquate. L'adaptation culturelle des outils de médiation ne vise en aucun cas à entretenir les préjugés à l'égard des différents membres du public, de leur origine ou de leur culture. Il s'agit bien au contraire d'assurer l'égalité et l'équité entre les visiteurs au sein des institutions patrimoniales : l'égalité, car le musée doit s'adresser à "tous" et que "tous" doivent avoir accès aux mêmes informations, et l'équité, pour que chacun puisse accéder à ces informations en fonction de ses connaissances et de ses compétences. Les principes fondamentaux de la médiation doivent donc être respectés, quel que soit le public considéré : ainsi, les textes doivent par exemple être lisibles et placés à proximité des œuvres qu'ils décrivent, ils doivent faciliter le jonglage objet-cartel ${ }^{7} . .$. Mais pour que "tous" puissent avoir accès au patrimoine exposé, les supports de médiation doivent prendre en compte les environnements de savoirs de chacun, qui peuvent diverger d'avec les œuvres et selon les visiteurs. Une aide à la visite pertinente doit donc être conçue en fonction non seulement de l'objet qu'elle décrit, mais aussi du public auquel elle s'adresse et de la culture de celui-ci. L'adaptation culturelle doit alors permettre de susciter les bons renvois intellectuels et les références, les connotations justes dans chaque langue et dans chaque culture afin de permettre la compréhension et l'immersion psychologique du plus grand nombre. 
Terrine canard, manufacture Chapelle (Sceaux), Cité de la Céramique (Sèvres/Limoges). Présentée à l'exposition Céramiques surprises. Faïences et trompe-l'œil des XVIII et XIXe siècles au musée départemental de la Faïence et des Arts de la table (Samadet). Il est parfois difficile de concevoir que certains objets nécessitent une "traduction culturelle", notamment lorsque les auteurs des outils de médiation baignent dans un environnement de savoirs spécifique. Nombre de Français verront dans cette terrine une note d'humour et un indice de l'importance de la table en France. Pourtant, des visiteurs issus d'autres référentiels culturels pourraient rencontrer quelques difficultés à apprécier que l'animal consommé soit représenté et exposé à table. Le musée n'a pas vocation à déterminer la légitimité de ces réactions, mais à dépasser celles-ci.

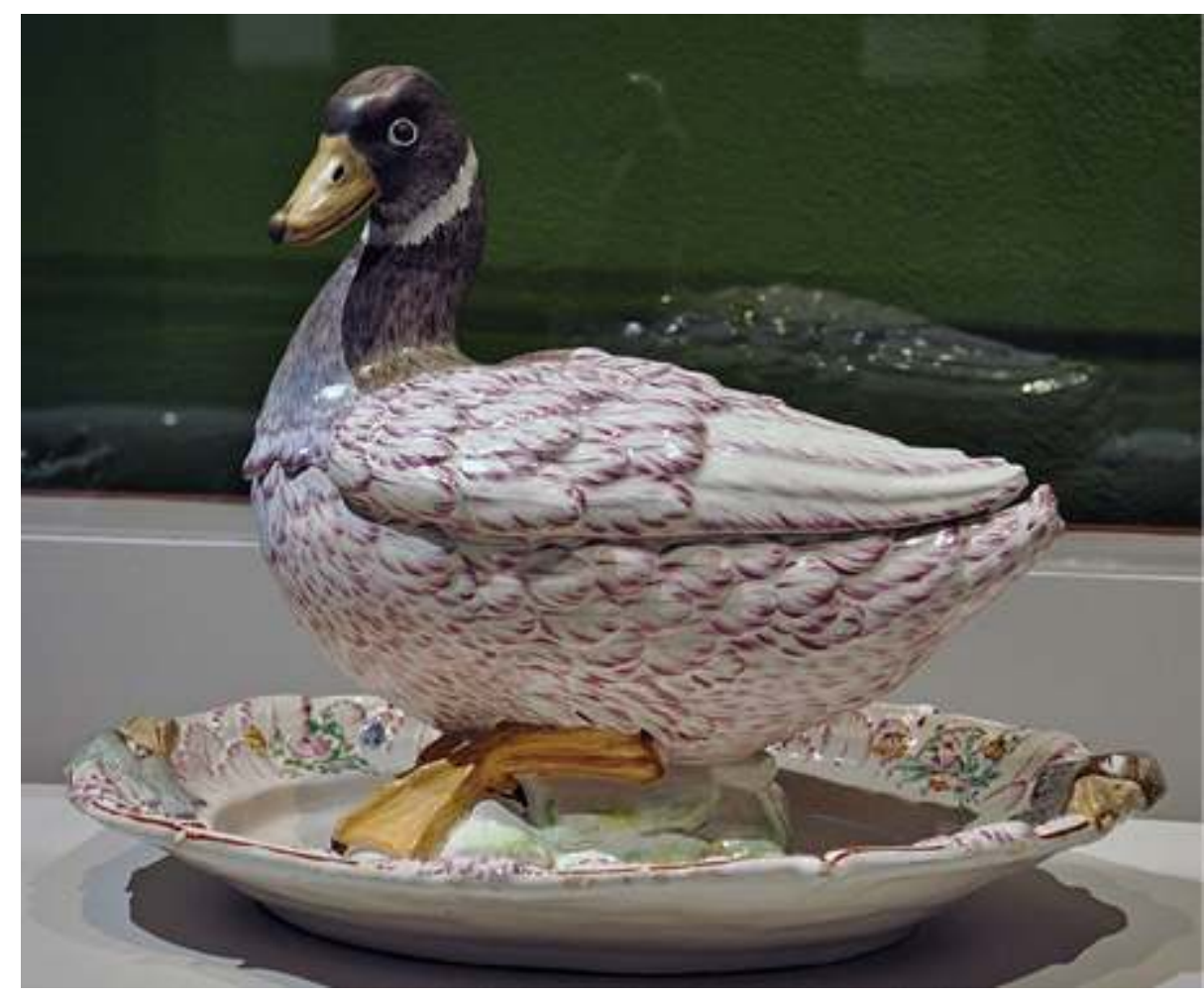

(c) Saoussan Sabeh

L'adaptation culturelle requiert subtilité et justesse dans son application, car elle ne doit pas devenir prétexte à la censure de certaines œuvres, qui aurait pour effet d'enfermer certains visiteurs dans la conception (fausse) qu'ils auraient du monde. Ainsi, pour certains publics, observer une nudité affichée, même à travers une œuvre d'art comme la Vénus de Milo, est inconvenant. Un guide chargé de faire découvrir l'histoire de l'art aux visiteurs ne peut cependant passer sous silence toutes les œuvres dévêtues qui peuplent nos musées. Pour beaucoup d'artistes, l'objectif n'était pas de donner à voir le nu pour le $\mathrm{nu}$, mais de représenter l'idéal, de correspondre aux codes iconographiques en vigueur à leur époque, ou simplement de choquer, en heurtant certaines mentalités. Expliquer les "dessous" d'une œuvre s'avèrerait bien plus enrichissant pour le public que d'entretenir ses tabous. Quand bien même l'œuvre ne plairait pas au visiteur, celui-ci aurait du moins fait la découverte de ses contextes et pourrait désormais justifier son opinion en toute connaissance de cause.

30 L'objectif véritable de l'adaptation culturelle consiste bel et bien à ouvrir de nouveaux horizons au public, tout en conservant la neutralité scientifique requise. Par neutralité scientifique et objectivité, il s'entend que les institutions muséales se devraient de s'attacher à présenter leurs expôts avec véracité, selon des informations exactes, des faits scientifiques, des vérités avérées, ou en expliquant clairement qu'elles ne sont pas en 
mesure de renseigner leurs visiteurs avec certitude, et qu'elles évoquent donc des hypothèses de recherche, des points de vue, auxquels le public peut adhérer ou non ; en résumé, les musées devraient continuer à transmettre l'état contemporain des connaissances au public.

80 \% des établissements sollicités se disent d'ailleurs attachés et fidèles au principe d'objectivité. Seuls $10 \%$ des institutions avouent choisir un référentiel culturel particulier : soit celui de leurs visiteurs, afin de ne pas heurter leur sensibilité, soit celui du territoire dans lequel elles s'inscrivent, par inadvertance ou pour plus de facilité. Les $10 \%$ restant font part du choix d'un référentiel particulier (artistique) dans le but avoué d'immerger le public dans l'esprit d'un artiste, d'un auteur ou d'un créateur. L'adaptation culturelle requiert une grande exigence de la part des musées, car le rattachement à un environnement de savoirs spécifique peut finalement s'avérer inconscient, justement parce qu'il appartient au référentiel culturel de l'institution patrimoniale considérée et de son personnel, qui n'y voient aucun positionnement puisqu'ils y baignent au quotidien. Ainsi, la "défaite" de Waterloo n'a ce titre qu'en France, puisqu'outre-Manche elle devient une "victoire". Le terme "bataille" s'avère alors plus judicieux, neutre et objectif.

L'odalisque, musée du Louvre (Paris). Alors que certains publics auraient besoin que leur soit expliquée cette volonté de Boucher de représenter une nudité dans semblable position, d'autres, déjà au fait des dessous d'une telle iconographie, pourraient en approfondir le contexte historique, par exemple.

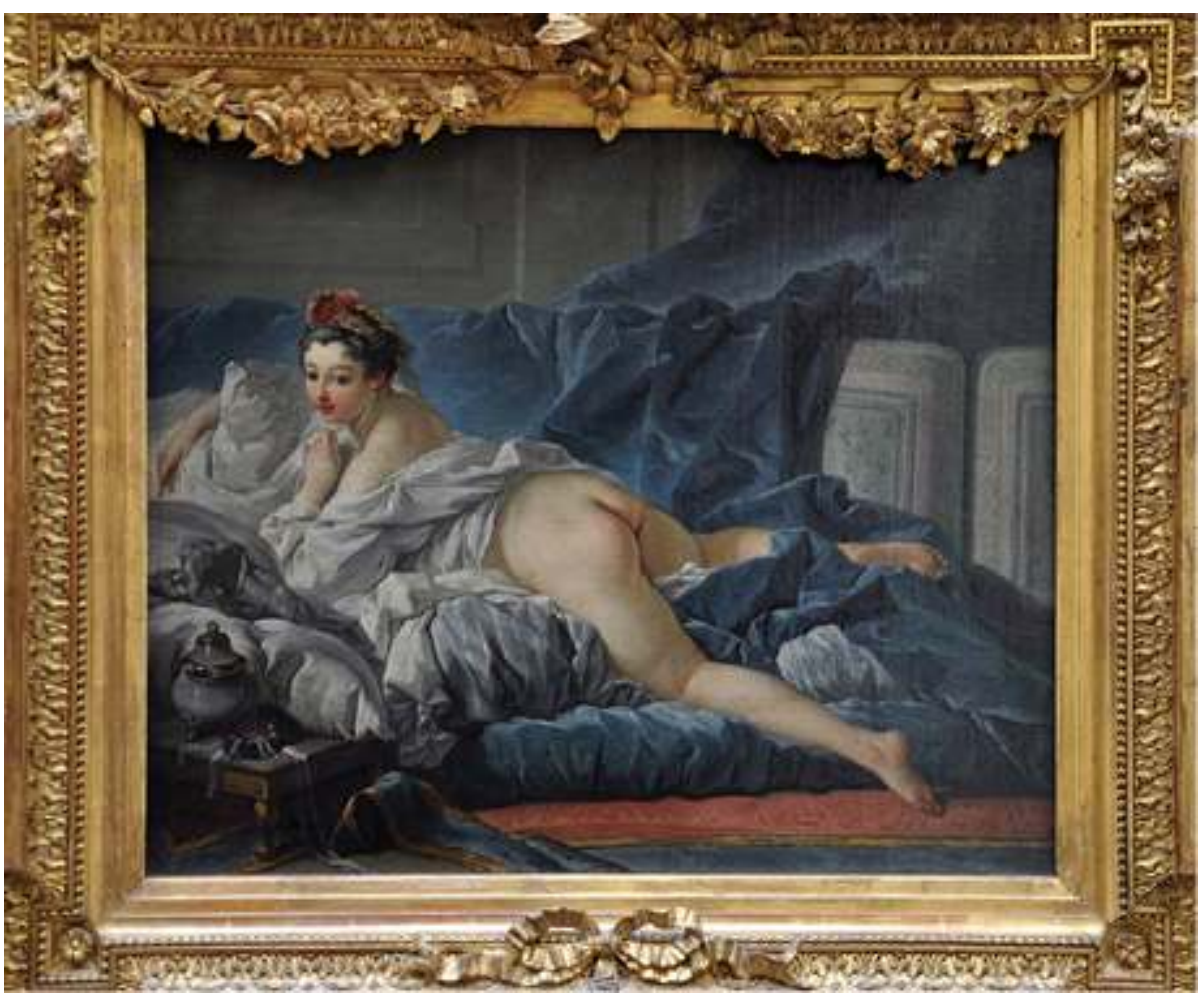

(C) Saoussan Sabeh

La difficulté de l'adaptation, outre qu'il faille bien sûr connaitre l'œuvre à décrire et le ou les publics auxquels s'adresser, demeure dans la modulation des données transmises aux visiteurs. Les supports de médiation sont limités, en volume et/ou en temps. Or, il semble préférable que tous les visiteurs bénéficient d'outils et de contenus équivalents (et non identiques, pour ce qui est des contenus) : il s'avèrerait en effet injuste et inégal que 
certains publics, parce qu'issus de telles ou telles cultures, dussent se contenter d'audioguides, ou de textes ardus, quand d'autres disposeraient de supports plus aisés, ou d'un véritable choix entre fiches de salle, audioguides, guides, tablettes...

Le défi de l'adaptation réside donc dans le fait de devoir, à travers un même support et une durée ou un volume équivalent, transmettre des indications similaires à tous, mais à des degrés différents, selon qu'il faille expliquer l'information "de surface" ou l'approfondir, en fonction de l'environnement de savoirs du public visé. En résumé, la profondeur des données varie en fonction des visiteurs considérés, tout comme le vocabulaire utilisé et la forme du discours, dont la structure est fonction du public auquel il s'adresse.

\section{Conclusion}

L'adaptation culturelle des aides à la visite se révèle très exigeante et délicate à mettre en œuvre. Pourtant, elle peut prendre des formes très simples et tout aussi satisfaisantes pour le public, en proposant par exemple des visites guidées en fonction des habitudes alimentaires (l'horaire du déjeuner peut différer de manière conséquente entre les pays considérés), ce qui permet une adéquation entre conservation, fréquentation et compréhension du patrimoine.

L'adaptation culturelle porte des enjeux d'une extrême importance pour la construction d'une société harmonieuse, riche de sa diversité et où l'obscurantisme n'aurait aucune prise.

\section{BIBLIOGRAPHY}

Charte européenne des langues régionales ou minoritaires, ouverte à la signature le 5 novembre 1992 à Strasbourg par le Conseil de l'Europe, et rapport explicatif. Consultable sur le site Internet du Conseil de l'Europe.

Cousin, S. L'identité au miroir du tourisme. Usages et enjeux des politiques de tourisme culturel. Thèse de doctorat, École des hautes études en sciences sociales, Paris, janvier 2003.

Dufresne-Tassé, C. L'exposition comme parcours de sens. Séminaire, Paris : École du Louvre, janvier 2016.

Grassin, A.-S. Le jonglage objet-cartel, La Lettre de l'Ocim, mars-avril 2007, n¹10, pp. 4-12.

Klein, M. et Yin, S. Adaptation au public français d'une exposition itinérante préparée par le musée du château Ramezay. Maîtrise de muséologie, Montréal : Université du Québec, 2005.

Les divers textes législatifs français existants relatifs à la défense, au développement et à la promotion des langues et des cultures régionales et minoritaires.

Mairesse, F. Géopolitique du musée. Séminaire, Paris : École du Louvre - Université Sorbonne Nouvelle-Paris 3, octobre 2015-janvier 2016. 
O'Neill, M.-C. L'exposition comme parcours de sens. Séminaire, Paris : École du Louvre, octobre à décembre 2015.

Rigat, F. Pourquoi et jusqu'où traduire les textes dans un musée et une exposition? La Lettre de l’Ocim, $\mathrm{n}^{\circ} 132$, novembre-décembre 2010, pp. 34-39.

Sabeh, S. Du multilinguisme au multiculturalisme : les enjeux de la diversité. Mémoire de recherche, Paris : École du Louvre, 2016.

Van Geert, F. Du musée colonial au musée des diversités. Intégrations et effets du multiculturalisme sur les musées ethnologiques. Thèse de doctorat, université de Barcelone, septembre 2014.

\section{NOTES}

1. Article $1^{\text {er }}$ de la loi $n^{\circ} 2002-5$ du 4 janvier 2002 relative aux musées de France, dite loi Musée : "Est considérée comme musée, au sens de la présente loi, toute collection permanente composée de biens dont la conservation et la présentation revêtent un intérêt public en vue de la connaissance, de l'éducation et du plaisir du public".

2. Enquête menée par l'auteur auprès de 62 institutions muséales, en France et à l'étranger. Pour plus de précisions concernant le cadre et les données de ces recherches, il est possible de contacter directement l'auteur (adresse électronique fournie en introduction) ou de consulter son ouvrage : Sabeh, S. Du multilinguisme au multiculturalisme : les enjeux de la diversité. Paris : École du Louvre, 2016, $184 \mathrm{p}$.

3. Les taux mentionnés doivent être entendus à titre indicatif, car les nombres de base pouvaient varier en fonction des contributions et des réponses apportées aux questionnaires.

4. À la rédaction de cet article en février 2017, le dernier texte officiel en la matière est celui de la proposition de loi relative à la promotion des langues régionales, adoptée par l'Assemblée nationale en première lecture le 31 janvier 2017 (texte adopté ${ }^{\circ} 897$ ).

5. Les statistiques établies lors de l'enquête dépendent des indications données par les établissements contributeurs. Certains musées ont ainsi fait part des deux réactions, énoncées simultanément par leur public (composé de visiteurs dont certains disposaient d'aides à la visite dans leur langue et d'autres non).

6. Là encore, certains musées ont mentionné les deux statuts dans leur réponse, ce qui explique les taux obtenus.

7. Voir à ce sujet l'article de Grassin, A.-S. Le jonglage objet-cartel, La Lettre de l'Ocim, n¹10, marsavril 2007, pp. 4-12.

\section{INDEX}

Mots-clés: Diversité culturelle, public, collections, musées 


\section{AUTHOR}

\section{SAOUSSAN SABEH}

diplômée de l'École du Louvre et auxiliaire documentaliste au musée des Beaux-Arts de Bayonne sabeh@orange.fr 